\title{
A Framework for Automatic Time-Domain Characteristic Parameters Extraction of Human Pulse Signals
}

\author{
Pei-Yong Zhang ${ }^{1}$ and Hui-Yan Wang ${ }^{2}$ \\ ${ }^{1}$ Institute of VLSI Design, Zhejiang University, Hangzhou 310027, China \\ ${ }^{2}$ College of Computer Science and Information Engineering, Zhejiang Gongshang University, Hangzhou 310018, China
}

Correspondence should be addressed to Hui-Yan Wang, cederic@mail.zjgsu.edu.cn

Received 21 May 2007; Revised 17 September 2007; Accepted 19 November 2007

Recommended by Tan Lee

A methodology for the automated time-domain characteristic parameter extraction of human pulse signals is presented. Due to the subjectivity and fuzziness of pulse diagnosis, the quantitative methods are needed. Up to now, the characteristic parameters are mostly obtained by labeling manually and reading directly from the pulse signal, which is an obstacle to realize the automated pulse recognition. To extract the parameters of pulse signals automatically, the idea is to start with the detection of characteristic points of pulse signals based on wavelet transform, and then determine the number of pulse waves based on chain code to label the characteristics. The time-domain parameters, which are endowed with important physiological significance by specialists of traditional Chinese medicine (TCM), are computed based on the labeling result. The proposed methodology is testified by applying it to compute the parameters of five hundred pulse signal samples collected from clinic. The results are mostly in accord with the expertise, which indicate that the method we proposed is feasible and effective, and can extract the features of pulse signals accurately, which can be expected to facilitate the modernization of pulse diagnosis.

Copyright (c) 2008 P.-Y. Zhang and H.-Y. Wang. This is an open access article distributed under the Creative Commons Attribution License, which permits unrestricted use, distribution, and reproduction in any medium, provided the original work is properly cited.

\section{INTRODUCTION}

Pulse diagnosis is one of the most important examinations. The doctors diagnose the patient by feeling the pulse beating at the measuring point of the radial artery, which requires long experiences and a high level of skill. Traditional pulse diagnosis is subjective and deficient in quantitative criteria of diagnosis. Therefore, quantitative methods are needed. Much effort is being spent on pulse analysis, such as the classification of pulse waveforms [1-7] and cardiovascular assistant diagnosis [8-11]. In pulse diagnosis, time-domain parameters can reflect the specificity of pulse signals. So they are endowed with important physiological significance by specialists of traditional Chinese medicine (TCM) and have obvious medical diagnostic importance [1]. A study on the construction of pulse diagnostic model based on timedomain characteristic parameters was done in [5], which demonstrated that time-domain characteristics can be representative of pulse signals. In a pioneer work, pulse signal characteristic points were mostly marked manually and the parameters were extracted by ocular estimation, which undoubtedly impeded the modernization of pulse diagnosis and its applications in clinic. Pulse signal is a kind of weak, nonstationary, low-frequency signal. An attractive tool for analyzing the local behavior of such signals is wavelet transform (WT), which can decompose signals into elementary building blocks that are well localized both in space and frequency $[12,13]$. Previous works, such as $[14,15]$, testify that wavelet transform is superior to frequency-domain analysis in the feature extraction of pulse waveform, but they deal only with the extraction of energy features. A paper relevant to time-domain feature extraction based on wavelet module maximum was published in [16], in which, the procedure of characteristic points labeling is very complex and not based on a mathematical theory. Besides, this method is only experimented on two pulse signal samples, which is not enough to validate the effectiveness of the proposed method. Another relevant published work based on wavelet transform was done in [17], in which, only wave crest and wave hollow can be detected. To date, none of the methods developed is perfect. In order to extract the parameters of pulse signals automatically, a new pulse characteristic detection approach 


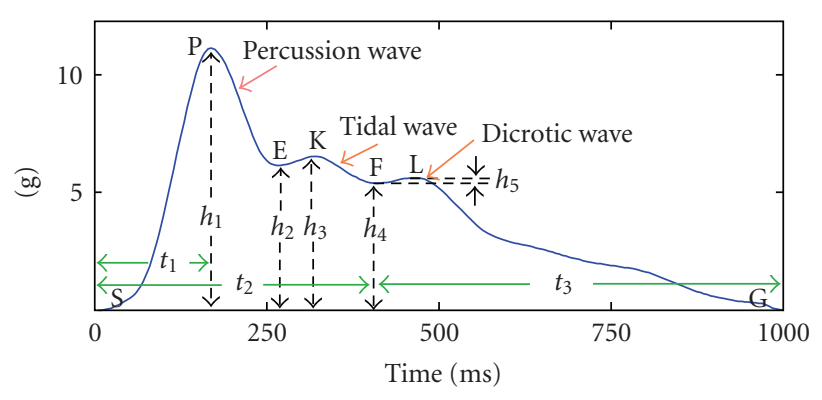

FIgURE 1: Time-domain parameters of pulse signal. This pulse signal sample is a triple-humped waveform, where S, P, E, K, F, and G are the characteristic points. The percussion wave, tidal wave, and dicrotic wave are three separate waves, on which the parameters are extracted. The $y$-axis is the amplitude of the pulse signal whose unit is gram force $(\mathrm{g})$. The $x$-axis is the time whose unit is millisecond.

based on complex-valued wavelet transform and chain code is proposed and tested in this work.

In this paper, a framework for automated parameter extraction of pulse signals is constructed. The framework includes characteristic point detection, estimation of the number of pulse waveform peak, labeling of characteristic points, and parameters computation. To validate the proposed methodology, a pulse signal database is used, in which, five hundred pulse signal samples and corresponding parameters computed manually by specialists are recorded from several hospitals.

This paper is organized as follows. The fundamental constituent of pulse signal, obtained through a pressure sensor, and the physiological significance of time-domain parameters are described in Section 2. The methodology of pulse parameter extraction is detailed in Section 3. The numerical experiments are reported in Section 4, followed by the conclusion in Section 5.

\section{TIME-DOMAIN CHARACTERISTIC PARAMETERS}

Figure 1 presents a period of a pulse waveform of a healthy volunteer, which is obtained by a pulse transducer. Figure 2 illustrates the pulse signal acquisition system. The sampling rate is $100 \mathrm{~Hz}$. The pulse transducer is belt-mounted and fixed on the radial pulse at the wrist while sampling pulse signal. This system can record a series of pulse signals under different contact pressures. The pulse signal whose modulus reaches the maximum is selected as the subject investigated. As the contact pressure of pulse transducer increases, the amplitude of the pulse signal first increases, reaching a maximum point, and then decreases. One period of pulse waveform is usually composed of three waves: a percussion wave, a tidal wave, and a dicrotic wave. The time-domain parameters, which have been testified to be important for diagnosis, are marked in Figure $1: h_{1}, h_{2}, h_{3}, h_{4}, h_{5}, t_{1}, t_{2}$ and $t_{3}$. These parameters all have an important physiological, pathological and psychological significance [1]. For example, the parameter $h_{1}$ is the amplitude of percussion wave, reflecting the ejection function of the left ventricle and the resilience of the main artery. The parameter $t_{1}$ presents the left ventricular ejection time. The parameters are computed based on the characteristic points, of which, $\mathrm{S}$ is the onset of percussion wave, $\mathrm{P}$ the peak of percussion wave, $\mathrm{E}$ the onset of tidal wave, $\mathrm{K}$ the peak of tidal wave, $\mathrm{F}$ the onset of dicrotic wave, and $\mathrm{L}$ the peak of dicrotic wave.

The pulse waveform is called a triple-humped wave (TRIWave), which has three peaks. The parameter can be marked as shown in Figure 1. However, for some pulse pattern, such as slippery pulse and wiry pulse, their pulse waveforms may have two peaks, which is called a double-humped wave (DOU-Wave). Figure 3 shows two Dou-Wave samples. The percussion wave of the slippery pulse is high, and the tidal wave superposes with a dicrotic wave. We named such pulse waveform as TDC-Wave, in which, the characteristic points $\mathrm{E}, \mathrm{K}$, and $\mathrm{F}$ are overlapping, and the parameters $h_{3}$ are equal to $h_{4}[1]$. For the wiry pulse, the percussion wave is round and broad and superposes with the tidal wave. Such pulse waveform is named as PTL-Wave, in which, the characteristic points $\mathrm{P}, \mathrm{E}$, and $\mathrm{K}$ are overlapping, and the parameters $h_{1}$ are equal to $h_{3}[1]$.

The above preliminary analysis has provided important information that the extraction of parameters not only needs to detect the characteristic points, but also requires to estimate the number of pulse waveform peaks, that is, to differentiate between TRI-Wave and DOU-Wave. If the pulse waveform is a DOU-Wave, it further needs to be classified into TDC-Wave and PTL-Wave.

\section{METHODOLOGY}

\subsection{Preprocessing of pulse signals}

Pulse signals can be easily contaminated by background noises, such as the uncontrollable movements of body limbs, respiration, and so on. Much work has been reported recently in pulse signal noise reduction [18] and baseline wander removal $[19,20]$, which have achieved good performance. In this research, the background noise and baseline wander are eliminated based on a decomposition and reconstruction algorithm of wavelet, which is similar to the work done in [20]. By simulation, we found that a smooth and symmetric wavelet function, such as the Mexican Hat wavelet [21], can remove noise effectively and preserve the important information of the pulse signal at the same time. In addition, we found that the optimum scale $a$ is equal to 4 . We choose the Mexican Hat wavelet and decompose the contaminated pulse signal to scale eight. Suppose $A_{4}$ and $A_{8}$ are the approximations of the Mexican Hat wavelet at fourth and eighth scale decompositions. Then $A_{8}$ is used to approximate the baseline wander of the pulse signal, and $A_{4}-A_{8}$ is just the pulse signal filtered. The reason that $A_{4}-A_{8}$ and $A_{8}$ are chosen as the approximation of pulse signal and its baseline wander can be seen in [20]. Figure 4 gives the filtered result of one contaminated pulse signal sample, which shows that the method we used to preprocess the pulse signal is effective. 

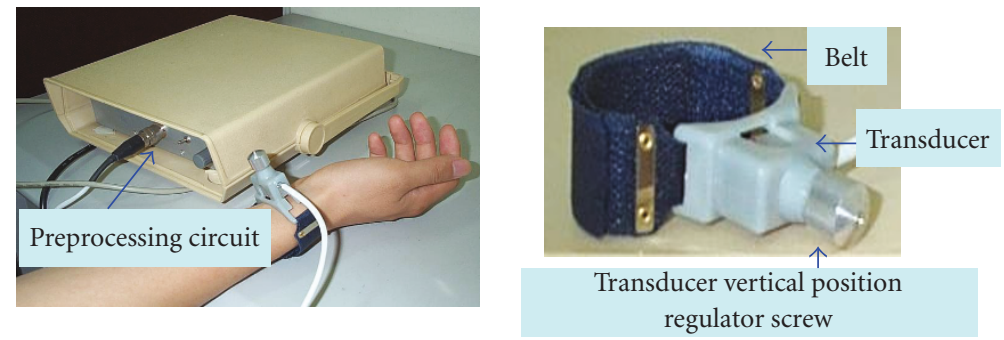

FIGURE 2: Pulse signal acquisition system, which consists of a preprocessing circuit and a pulse transducer. The preprocessing circuit is comprised of two amplifiers and an analog-digital converter. The transducer is made by Shanghai University of Traditional Chinese Medicine, Shanghai, China. It is a duplex cantilever beam transducer, which can be distinguished from sensors used in western medicine. The sensitivity and output impedance are 0.5 millivolt per gram $(\mathrm{g})$ and one thousand ohm, respectively. The output dynamic range of the pulse signal acquisition system is from zero to fifty gram force $(\mathrm{g})$.

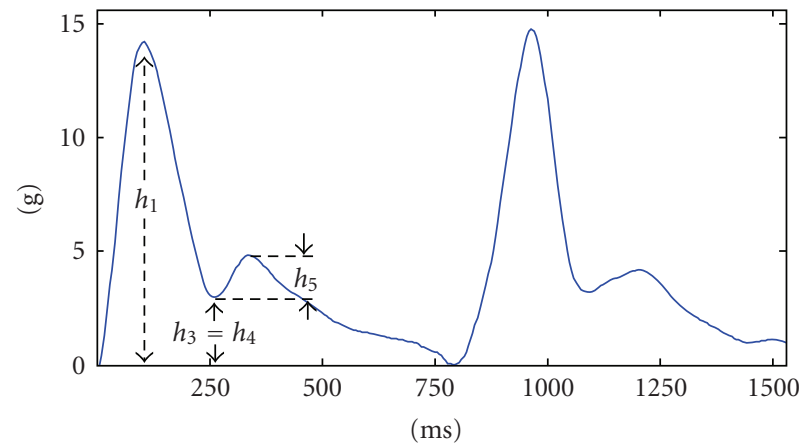

(a)

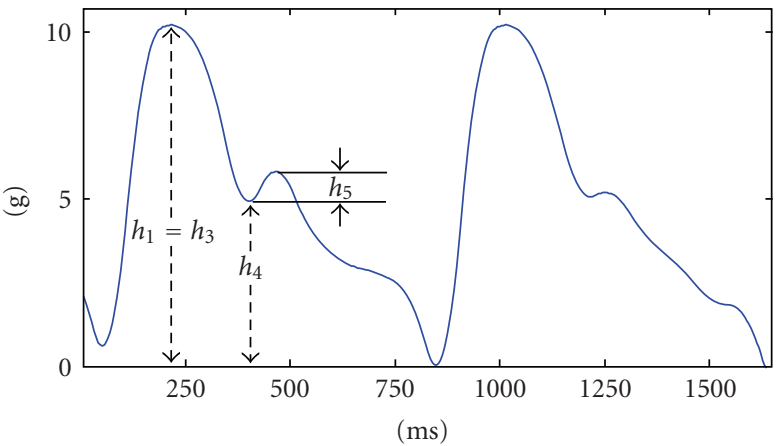

(b)

FIGURE 3: A slippery pulse sample which is a TDC-Wave, a wiry pulse sample which is a PTL-Wave, and some of the labeling of their parameters.

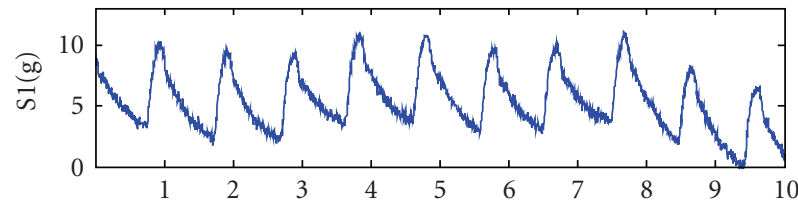

(S)

(a)

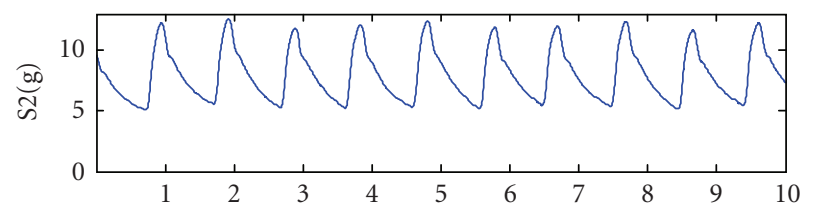

(S)

(b)

Figure 4: A contaminated pulse signal sample and its preprocessed result. $\mathrm{S} 1$ is a pulse signal sample contaminated with background noise and baseline wander. S2 is the result of S1 filtered with wavelet filter.

\subsection{Detection of characteristic points}

The pulse parameters are computed based on the corners of pulse signals [1]. Corners are locations where the curva- ture changes sharply, regarded as the most descriptive features, and can be characterized by the modulus of their wavelet transforms [22]. Recently, several corner detection techniques based on WT [22-24] have been developed and applied in some domains, such as object recognition [25]. In this study, the characteristic points of pulse signals are detected based on complex-valued wavelet transform, which is testified to be more effective than methods based on realvalued wavelet transform by our experiments.

Let $\psi(x)$ be a complex-valued wavelet, the continuous wavelet transform of the pulse signal $f(x)$ with respect to the wavelet $\psi(x)$ is defined as

$$
W f(a, b)=\int_{-\infty}^{+\infty} f(x) \overline{\psi_{a}}(x-b) d x
$$

where $\psi_{a}(x)=1 / a \psi(x / a)$ and $\overline{\psi_{a}}(x)$ denotes the complex conjugate of $\psi_{a}(x)$.

We choose the second derivative of the Gaussian function $\theta_{\sigma}(x)$, which has two vanishing moments [13], as the realvalued wavelet $\psi^{r}(x)$,

$$
\psi_{\sigma}^{r}(x)=-\frac{d^{2} \theta_{\delta}(x)}{d x^{2}}
$$


The modulus maxima of the wavelet transform correspond to the curvature of high order. Then the real wavelet transform of $f(x)$ is written as

$$
W f^{r}=f(x) * \psi_{\sigma}^{r}(x)=\sigma^{2} f(x) * \frac{d^{2} \theta_{\delta}(x)}{d x^{2}} .
$$

We turn the real-valued wavelet $\psi^{r}(x)$ into the complexvalued wavelet $\psi(x)$ by means of Hilbert transform $H$ [26] as follows:

$$
\psi(x)=(1+i H) \psi_{\sigma}^{r}(x) .
$$

The frequency response of $\psi(x)$ is expressed as

$$
\hat{\Psi}(\xi)=K_{0} \xi^{2} e^{-\xi^{2} / 2} \chi_{(0, \infty)}(\xi),
$$

where $\chi_{(0, \infty)}(\xi)$ denote the Heaviside step function, which is equal to 1 when $\xi>0$ and to 0 otherwise. $K_{0}$ denote a normalization constant.

Let $\psi(x)=\psi^{r}(x)+j \psi^{i}(x)$, whose real part is shown as (3). By (4), we obtain the imaginary part as follows:

$$
\psi^{i}(x)=-\frac{1}{\pi} \int_{-\infty}^{+\infty} \frac{\sigma^{2}\left(d^{2} \theta_{\sigma} / d t^{2}\right)}{x-t} d x .
$$

By (6), the following equation can be inferred:

$$
\psi^{i}(x)=\frac{1}{\pi}\left(\sqrt{2 \pi} x-\theta_{\sigma}^{\prime \prime \prime}(x)+2 \theta_{\sigma}^{\prime}(x)\right) .
$$

Then the complex wavelet transform of $f(x)$ is

$$
\begin{aligned}
W f^{c}= & f * \psi_{\sigma}(x)=\sigma^{2} f(x) * \theta_{\sigma}^{\prime \prime}(x) \\
& +j \frac{-1}{\pi}\left(f *\left(\sigma^{3} \theta_{\sigma}^{\prime \prime \prime}(x)+2 \sigma \theta_{\sigma}^{\prime}(x)-\sqrt{2 \pi} \frac{x}{\sigma^{2}}\right)\right) .
\end{aligned}
$$

Suppose $\operatorname{Re}\left(f * \psi_{\sigma}(x)\right)$ is the real part of $W f^{c}$, and $\operatorname{Im}\left(f * \psi_{\sigma}(x)\right)$ the imaginary part. The wavelet modulus maxima can be found by

$$
\begin{gathered}
\frac{d\left|W f^{c}\right|^{2}}{d x}=2\left[\operatorname{Re}\left(W f^{c}\right) * \operatorname{Re}\left(W f^{c}\right)^{\prime}+\operatorname{Im}\left(W f^{c}\right)\right. \\
\left.* \operatorname{Im}\left(W f^{c}\right)^{\prime}\right]=0 .
\end{gathered}
$$

The corners of the pulse signal correspond to these modulus maxima. Figure 5 shows a sample of pulse signal and corresponding complex-valued wavelet transforms computed according to (8). The zero crossing points of $W f^{c}$ correspond to the corners of $f(x)$, that is, the characteristic points of the pulse signal.

\subsection{Estimation of the number of pulse waveform peaks}

Chain code is used to estimate the number of pulse waves in this study. The chain code is an algorithm that gives a symbolic representation of an object boundaries using a series of specific directional, straight, connected lines [27]. The common representation of chain code is based on an eightway directional system, whose definition of eight directions is shown in Figure 6. Typically, the numbering scheme of chain code is defined as $i=\{-3,-2,-1,0,1,2,3\}$. Because there are no loop curves in pulse waveform, the directions -3 and 3 are not included. Therefore, the value span of chain code can be expressed as $\{-2,-1,0,1,2\}$, where the number 0 represents east, 1 is northeast, 2 is north, 3 is northwest, and so on. Split the pulse waveform into $N_{t}$ segments. Let $d$ denote the length of each segment, and let $\theta_{d t}$ represent the separation angle between each segment and the $x$ axis. The value of chain code $V_{d}$ can be defined as

$$
V_{d}= \begin{cases}-2, & -90^{\circ} \leq \theta_{d t}<-67.5^{\circ} \\ -1, & -67.5 \leq \theta_{d t} \leq-22.5^{\circ} \\ 0, & -22.5^{\circ}<\theta_{d t}<22.5^{\circ} \\ 1, & 22.5^{\circ} \leq \theta_{d t} \leq 67.5^{\circ} \\ 2, & 67.5^{\circ}<\theta_{d t} \leq 90^{\circ} .\end{cases}
$$

Let $L_{d}$ denote the chain code string of a period of pulse waveform. We predict the number of peaks based on the fact that the values of chain code in $L_{d}$ jump from a positive number or zero to a negative number gradually when there is a peak in pulse waveform. The graphical representation of $L_{d}$ is some saw-tooth, square waves, and the trailing edges correspond to the peaks of pulse waveform. The steps involved in this approach are summarized as follows.

(1) Find the positions $p_{i}$ in $L_{d}, i=1,2, \ldots, C_{d}$, where the chain code drifts clockwise from one direction to another. Compute the length of the substring of chain code in both directions at $p_{i}$, denoted as $h_{i 1}$ and $h_{i 2}$, respectively.

(2) Let $\min h_{i}$ denote the minimum of $h_{i 1}$ and $h_{i 2}, i=$ $1,2, \ldots, C_{d}$. Set the length threshold to $T_{d}$ and count the number of $\min h_{i}$, which satisfies $\min h_{i}>T_{d}$, denoted as $N_{f}$. Then $N_{f}$ is just the number of peaks to be estimated. Figure 7 shows the chain code graphical representation of a DOUWave sample and a TRI-Wave sample. The length threshold $T_{d}$ is set to 5 and the parameter $d$ is set to $2 . N_{f}$ is estimated to be 2 in the former pulse waveform and 3 in the latter one, respectively.

\subsection{Characteristic points labeling}

To compute the time-domain parameters, the characteristic points detected need to be labeled. This can be accomplished by splitting the pulse signal into periodic components and computing the apex angle $\alpha$ of the pulse waveform. The determination of periodicity of pulse signal can be achieved by detecting the modulus maximum of pulse waveform based on wavelet transform [22]. For pulse signal, whose rhythm is normal, the distance between two adjacent modulus maxima is just one periodicity. The modulus maximum corresponds to the peak of percussion wave $\mathrm{P}$, and the characteristic point before $\mathrm{P}$ is labeled as the onset of percussion wave $\mathrm{S}$. If the pulse waveform is a TRI-Wave, the four characteristic points after $\mathrm{P}$ are labeled as the onset of tidal wave $\mathrm{E}$, the peak of 


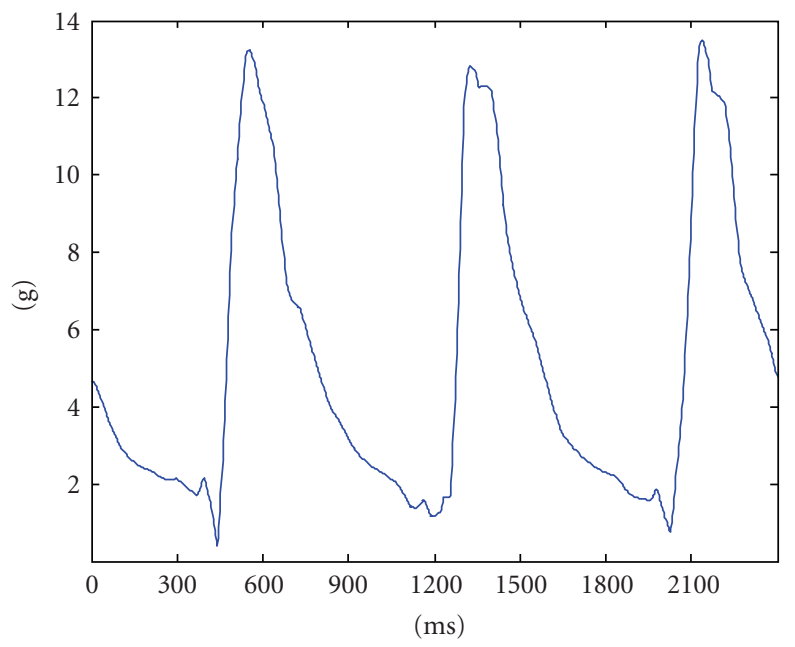

(a)

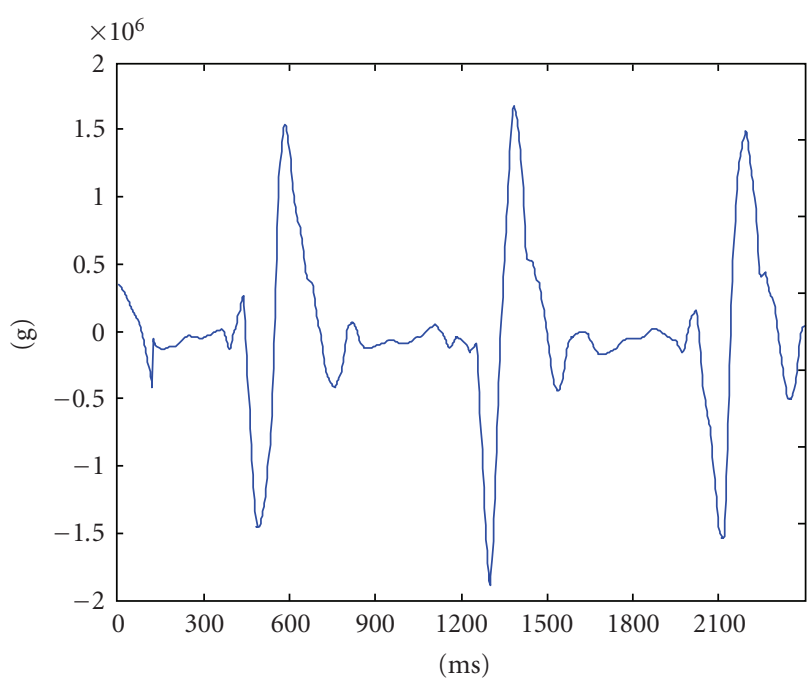

(b)

FIgURE 5: A pulse signal sample $f(x)(\mathrm{a})$, and the complex-valued wavelet transform $W f^{c}(\mathrm{~b})$.

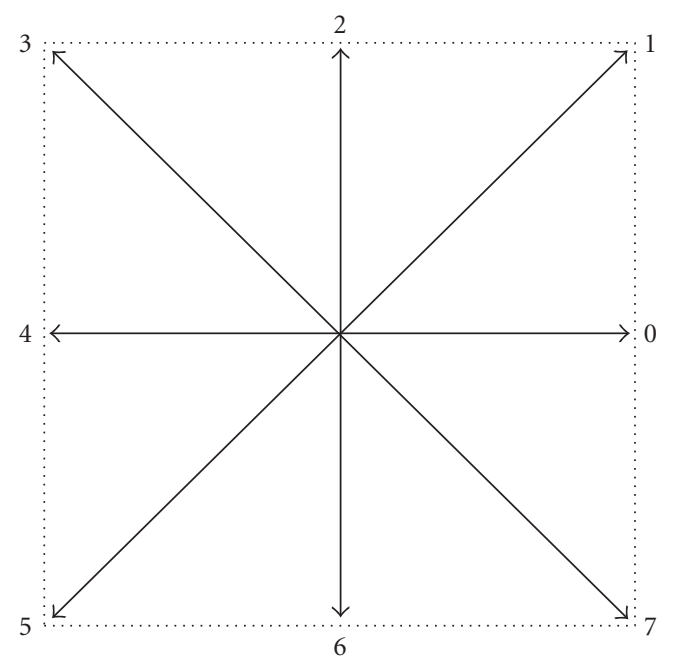

(a)

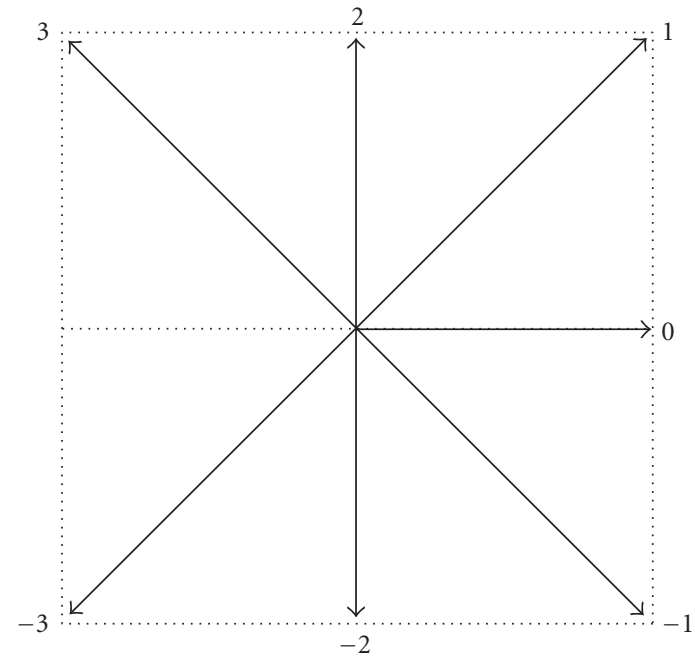

(b)

FIGURE 6: Eight connectivity directions and their numbering schemes (a), and the common chain code directions and their numbering scheme (b).

tidal wave $\mathrm{K}$, the onset of dicrotic wave $\mathrm{F}$, and the peak of dicrotic wave L, respectively, as shown in Figure 8(a).

On the basis of the concept mentioned above, DOUWave can be classified into TDC-Wave and PTL-Wave, in which, the characteristic points need to be labeled differently. For TDC-Wave, the percussion wave is high, steep, and thin, that is, the peak width is small. For PTL-Wave, the percussion wave is flat and broad or round and broad, that is, the peak width is large. We can differentiate TDC-Wave from PTLWave by computing the apex angle $\alpha$ of percussion wave, which can reflect the size of peak width. Suppose the coordinate of the peak of percussion wave $\mathrm{P}$ is $(x, f(x))$. Let $\mathrm{P}_{1}$ and $\mathrm{P}_{2}$ be points whose coordinates are $(x-n, f(x-n))$ and $(x+n, f(x+n))$. In this research, $n$ is set to 4 . The included angles between the longitudinal axis and $\mathrm{PP}_{1}$ and $\mathrm{PP}_{2}$ are denoted as $\alpha_{1}$ and $\alpha_{2}$, respectively, where $\operatorname{tg} \alpha 1=n / l_{1}$, $\operatorname{tg} \alpha 2=n / l_{2}, l_{1}=f(x)-f(x-n)$, and $l_{2}=f(x)-f(x+n)$. Then the apex angle $\alpha$ can be denoted as

$$
\operatorname{tg} \alpha=\frac{\operatorname{tg} \alpha 1+\operatorname{tg} \alpha 2}{1-\operatorname{tg} \alpha \operatorname{tg} \alpha 2}=\frac{n / l_{1}+n / l_{2}}{1-n^{2} / l_{1} l_{2}}=\frac{l_{2}+l_{1}}{l_{1} l_{2} / n-n} .
$$

Set threshold of $\alpha$ to $T_{\alpha}$. If $\operatorname{tg} \alpha>T_{\alpha}$, then the pulse waveform is a PTL-Wave. Otherwise, the pulse waveform is a TDC-Wave. For TDC-Wave, the two characteristic points after $\mathrm{P}$ are labeled as $\mathrm{E}(\mathrm{K}, \mathrm{F})$ and $\mathrm{L}$, as shown in Figure 8(b). For PTL-Wave, the characteristic points $\mathrm{P}, \mathrm{E}$, and $\mathrm{K}$ are overlapping, and the two characteristic points after $\mathrm{P}$ are labeled as $\mathrm{F}$ and $\mathrm{L}$, respectively, as shown in Figure 8(c). 

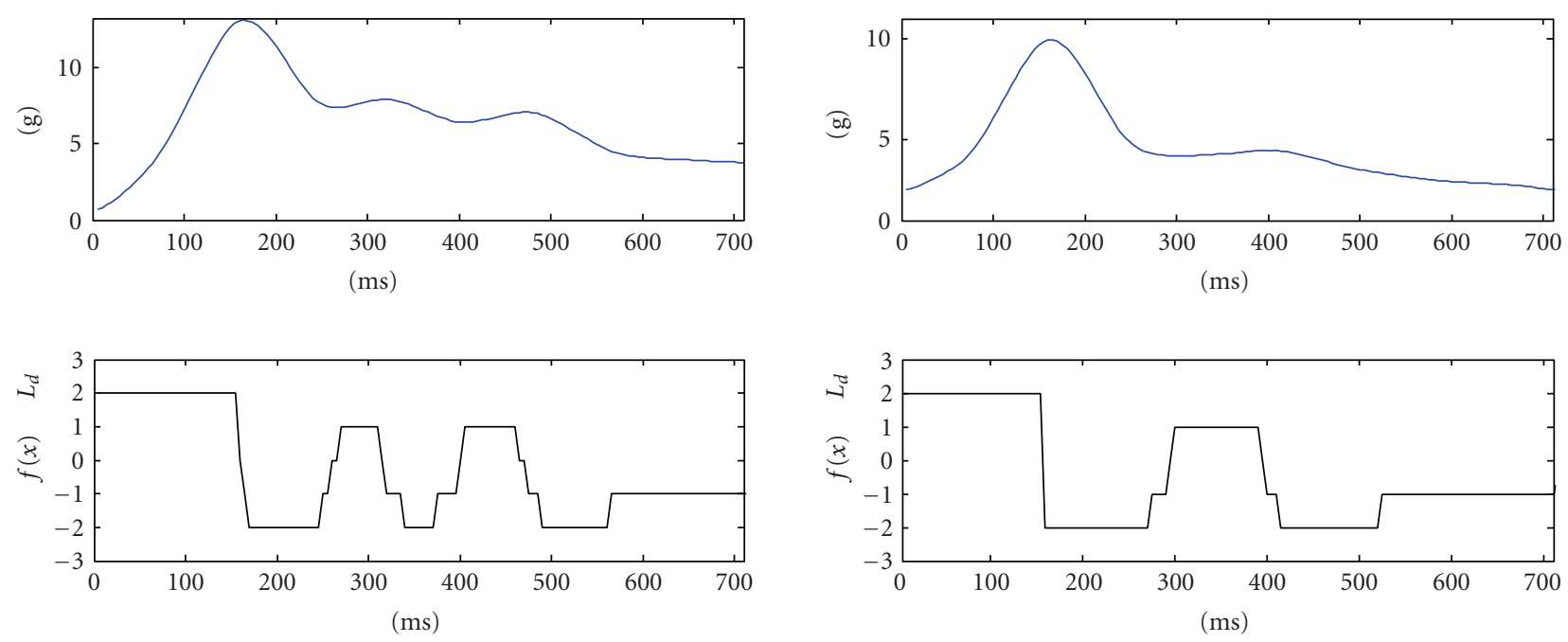

(a)

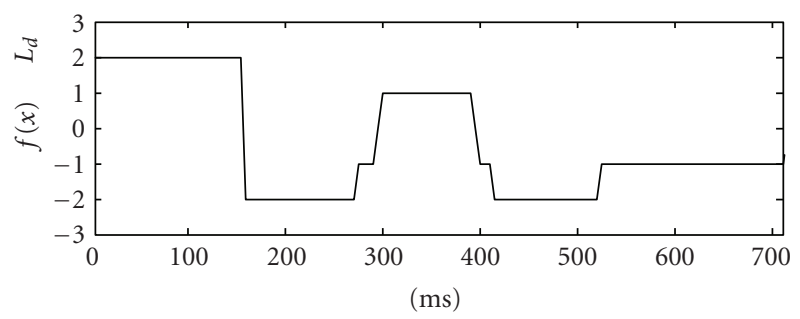

(b)

FIGURE 7: A period of a DOU-Wave sample and its graphical representation of chain code string (a), and a period of a TRI-Wave sample and its graphical representation of chain code string (b).

\section{EXPERIMENTAL RESULT}

To analyze the performance of the methodology we proposed, a pulse signal database is employed, in which a total of 500 pulse signals were gathered from several hospitals of TCM. The experiments will verify four objectives: (1) the proposed approach can detect the characteristics of the pulse signals accurately, and it is superior to the conventional techniques based on real-valued wavelet transform; (2) the proposed approach can estimate the number of pulse waveform peaks correctly, that is, the pulse waveform can be classified into TRI-Wave and DOU-Wave effectively; (3) the proposed approach can discriminate between TDC-Wave and PTL-Wave accurately; (4) the parameters can be estimated precisely based on the proposed method.

In experiment I, we draw a comparison between the real-valued wavelet and the proposed method to validate the first objective, which is shown in Figure 9. Firstly, the background noise is removed by selecting the Mexican hat wavelet as the filter and setting a scale equal to 4 . Secondly, the characteristics are detected, shown by "*." By simulation, we found that the same characteristics are detected for all modulus thresholds based on complex-valued wavelet [26], which is depicted as Figure 9(b). Figure 9(c) is a one-periodic time intercept of Figure 9(b), which is labeled based on a proposed method. Figures 9(d) and 9(e) show that the corners detected based on real-valued wavelet are too many to be labeled. The reason is that the modulus of real-valued wavelet is oscillatory [13]. Figure 9(f) illustrates that the number of characteristics is near to six, but the position is located incorrectly when modulus threshold is set to twenty. This experiment testifies that the proposed algorithm outperforms the conventional real-valued wavelet-based method in the characteristic detection of pulse signals.
TABLE 1: PAR results.

\begin{tabular}{lcccc}
\hline $\begin{array}{l}\text { Pulse } \\
\text { waveform }\end{array}$ & TRI-Wave & DOU-Wave & TDC Wave & PTL Wave \\
\hline PAR $(\%)$ & 97.8 & 95.6 & 96.9 & 94.9 \\
\hline
\end{tabular}

TABLE 2: Confusion matrix of DOU-Wave and TRI-Wave.

\begin{tabular}{lcc}
\hline & DOU-Wave & TRI-Wave \\
\hline DOU-Wave & 262 & 12 \\
TRI-Wave & 5 & 221 \\
\hline
\end{tabular}

In experiment II, the second and the third objectives are validated. The predictive accuracy rate (PAR) is computed, which is defined as

$$
\text { PAR }=\frac{\text { number of samples correctly classified }}{\text { total number of samples }} .
$$

The results using PAR are presented in Table 1. For the convenience of illustration, the confusion matrix of DOU-Wave and TRI-Wave is shown in Table 2, and the confusion of TDC-Wave and PTL-Wave is given in Table 3. The PAR of TRI-Wave is $97.8 \%$ and five samples were misclassified. We found that if the tidal wave is low and near to dicrotic wave, the TRI-Wave is prone to be mistaken for DOU-Wave. The PAR of DOU-Wave is $95.6 \%$ and twelve samples, which have interfering small waves, were mistaken for TRI-Wave. For TDC-Wave and PTL-Wave, the PAR are 96.9\% and 94.9\%, respectively. We found that if the shape of percussion wave is irregular, such as forked, TDC-Wave is apt to be confounded with PTL Wave. 


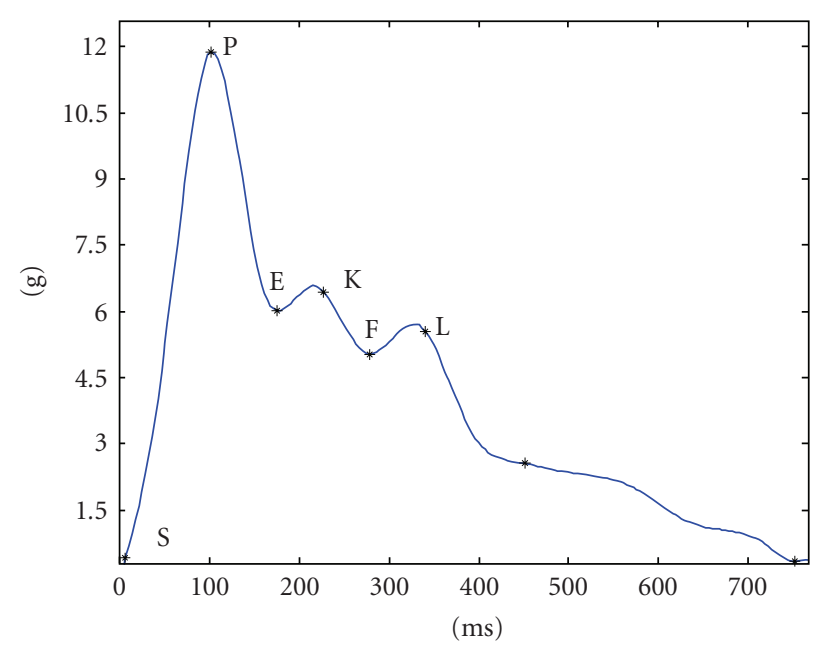

(a)

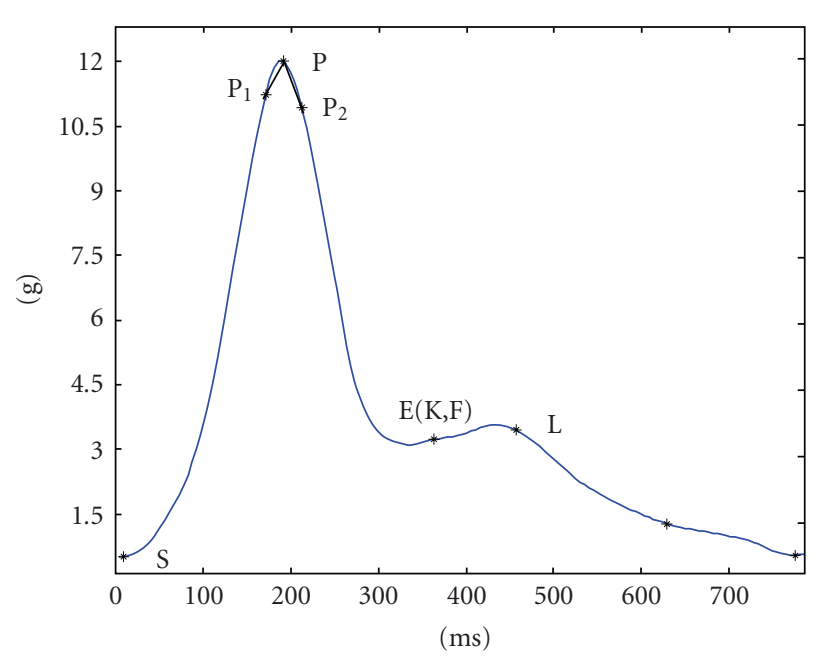

(b)

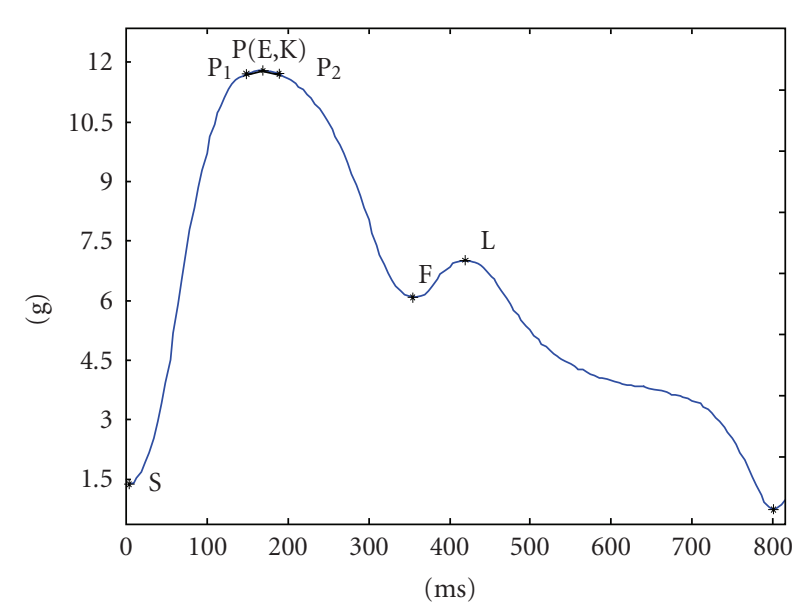

(c)

FIGURE 8: Detection result and labeling of characteristic points of a TRI-Wave (a), characteristic points detecting and labeling of a TDC-Wave where $\alpha=5.8^{\circ}(\mathrm{b})$, and characteristic points detecting and labeling of a PTL-Wave where $\alpha=122.8^{\circ}$ (c).
TABLE 3: Confusion matrix of TDC-Wave and PTL-Wave.

\begin{tabular}{lcc}
\hline & TDC-Wave & PTL Wave \\
\hline TDC-Wave & 94 & 3 \\
PTL-Wave & 9 & 168 \\
\hline
\end{tabular}

To explore the fourth objective, we design experiment III. Firstly, we label the characteristic points manually and compute the parameters according to these characteristic points. The parameter set is denoted as $P_{M}=\left\{P_{M_{1}}, P_{M_{2}}, \ldots, P_{M_{N}}\right\}$, where $P_{M_{I}}=\left\{h_{M 1_{i}}, h_{M 2_{i}}, h_{M 3_{i}}, h_{M 4_{i}}, h_{M 5_{i}}, t_{M 1_{i}}, t_{M 2_{i}}, t_{M 3_{i}}\right\}, 1 \leq$ $i \leq N$ and $N=500$, is the number of pulse samples. Secondly, we calculate the parameters based on the proposed methodology, and the parameter set is presented as $P_{U}=$ $\left\{P_{U_{1}}, P_{U_{2}}, \ldots, P_{U_{N}}\right\}$, where $P_{M_{I}}=\left\{h_{U 1_{i}}, h_{U 2_{i}}, h_{U 3_{i}}, h_{U 4_{i}}, h_{U 5_{i}}\right.$, $\left.t_{U 1_{i}}, t_{U 2_{i}}, t_{U 3_{i}}\right\}$. Thirdly, the mean square error function is modified and acts as the measurement of the difference between $P_{M}$ and $P_{U}$, which is defined as

$$
\operatorname{MSE}=\frac{\sqrt{\sum_{i=1}^{N}\left(x_{i}-x_{i 0}\right)^{2}}}{N},
$$

where $x_{i} \in P_{U_{i}}, x_{i 0} \in P_{M_{i}}$, and $x_{i}$ and $x_{i 0}$ correspond to the same parameter. Fourthly, MSE are estimated from all samples, which is indicated as MSE-ERR. Finally, the samples misclassified in experiment II are eliminated, and MSE are calculated based on the residual samples, which is denoted as MSE-COR. On the basis of discussions above, if the characteristic points of the pulse signals are detected correctly, then MSE-COR is low and vice versa. The MSE results are presented in Table 3. MSE-COR of the eight parameters are low and MSE-ERR of parameters $h_{2}, h_{3}, h_{4}, h_{5}, t_{2}$, and $t_{3}$ are relatively high. For parameters $h_{1}$ and $t_{1}$, MSE-COR and MSEERR are identical because they are not influenced by the classification of pulse waveform. The parameters $h_{2}$ and $h_{3}$ may be computed by mistake when TDC-Wave and PTL-Wave are misclassified. While the recognition of TRI-Wave and DOUWave is inaccurate, the computation of the parameters $h_{4}, h_{5}$, $t_{2}$ and $t_{3}$ would be in error. This experiment validate that the characteristic points are detected effectively and the parameters are computed accurately.

\section{CONCLUSIONS}

A methodology for characteristic parameters extraction of pulse signal is proposed. Starting from the detection of the characteristic points based on wavelet transform and then the number of pulse waveform peaks is determined using chain code. Based on the characteristic points labeled, the parameters are computed. The methodology has been tested using a pulse signal database. The results show that our method is feasible and effective. It can extract the parameters accurately, which could be expected to be useful in promoting the modernization of pulse diagnosis in the near feature. 


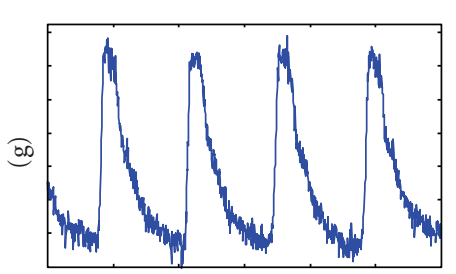

(ms)

(a)

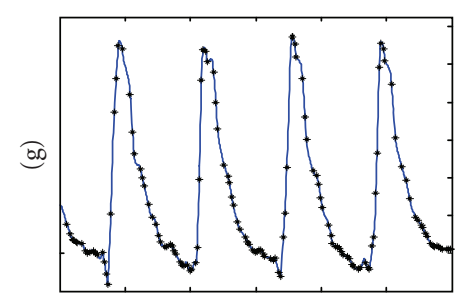

(ms)

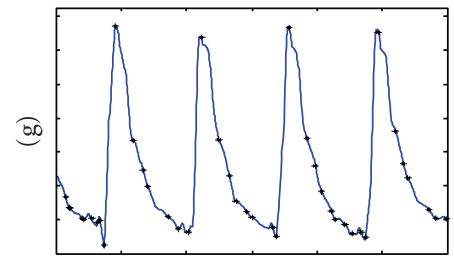

(ms)

(b)

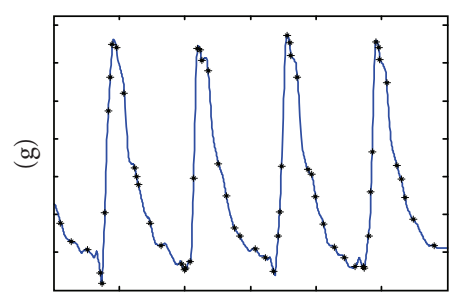

(ms)

(e)

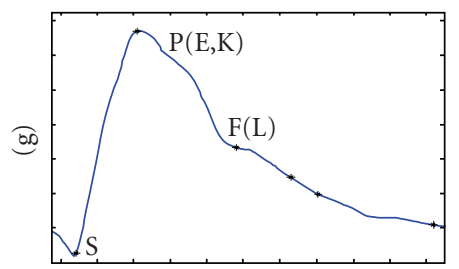

(ms)

(c)

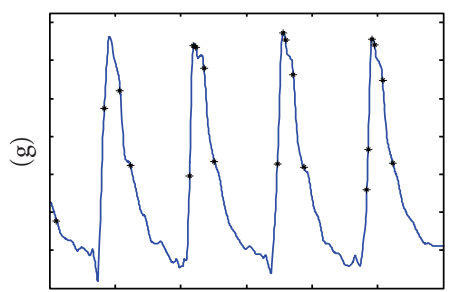

(ms)

(f)

FIGURE 9: A PTL-Wave pulse signal sample, which is contaminated by background noise (a); characteristic points detection result based on a complex-valued wavelet (b); labeling result based on a proposed method (c); characteristic points detection results based on real-valued wavelet when modulus threshold is set to zero (d), ten (e), and twenty (f), respectively.

TABLE 4: The MSE results of the parameters with the samples misclassified eliminated (MSE-COR) and the samples misclassified included (MSE-ERR).

\begin{tabular}{lcccccccc}
\hline Characteristic parameters & $h_{1}$ & $h_{2}$ & $h_{3}$ & $h_{4}$ & $h_{5}$ & $t_{1}$ & $t_{2}$ & $t_{3}$ \\
\hline MSE-COR & 0.3235 & 0.2363 & 0.2988 & 0.2096 & 0.1625 & 0.1245 & 0.1383 & 0.1184 \\
MSE-ERR & 0.3235 & 2.0139 & 2.5301 & 1.3209 & 0.9531 & 0.1245 & 0.8755 & 1.0627 \\
\hline
\end{tabular}

\section{APPENDIX}

\section{THE ALGORITHM DESCRIPTION OF AUTOMATIC TIME-DOMAIN CHARACTERISTIC PARAMETERS EXTRACTION}

The proposed algorithm is executed as follows.

(1) Perform the preprocessing algorithm based on wavelet decomposition and reconstruction to remove noise and baseline wander of pulse signals.

(2) Detect the characteristic points of pulse signals based on complex-valued wavelet.

(3) Estimate the number of pulse waveform peaks based on chain code.

(4) If the pulse signal is a DOU-Wave, compute the apex angle $\alpha$, according to which DOU-Wave is classified as TDC-Wave and PTL-Wave.

(5) Label the characteristic points of the pulse signals and compute the parameters.

\section{REFERENCES}

[1] Z. F. Fei, Contemporary Sphygmology in Traditional Chinese Medicine, People's Medical Publishing House, Beijing, China, 2003.
[2] M. F. Abbod, D. G. von Keyserlingk, D. A. Linkens, and M. Mahfouf, "Survey of utilisation of fuzzy technology in medicine and healthcare," Fuzzy Sets and Systems, vol. 120, no. 2, pp. 331-349, 2001.

[3] Y.-Z. Yoon, M.-H. Lee, and K.-S. Soh, "Pulse type classification by varying contact pressure," IEEE Engineering in Medicine and Biology Magazine, vol. 19, no. 6, pp. 106-110, 2000.

[4] L. Xu, D. Zhang, K. Wang, and L. Wang, "Arrhythmic pulses detection using Lempel-Ziv complexity analysis," EURASIP Journal on Applied Signal Processing, vol. 2006, Article ID 18268, 12 pages, 2006.

[5] H. Wang and Y. Cheng, "A quantitative system for pulse diagnosis in traditional Chinese medicine," in Proceedings of the 27th Annual International Conference of the IEEE Engineering in Medicine and Biology Society (EMBS '05), vol. 7, pp. 56765679, Shanghai, China, September 2005.

[6] L. S. Xu, K. Q. Wang, and L. Wang, "Pulse waveforms classification based on wavelet network," in Proceedings of the 27th Annual International Conference of the IEEE Engineering in Medicine and Biology Society (EMBS '05), vol. 7, pp. 45964599, Shanghai, China, September 2005.

[7] L. Wang, K.-Q. Wang, and L.-S. Xu, "Recognizing wrist pulse waveforms with improved dynamic time warping algorithm," in Proceedings of the 3rd International Conference on Machine Learning and Cybernetics (ICMLC '04), vol. 6, pp. 3644-3649, Shanghai, China, August 2004.

[8] K. Wang, L. Xu, Z. Li, D. Zhang, N. Li, and S. Wang, "Approximate entropy based pulse variability analysis," in Proceedings of 
the 16th IEEE Symposium on Computer-Based Medical Systems (CBMS '03), pp. 236-241, New York, NY, USA, June 2003.

[9] Y. Diao, S. C. Wu, Y. J. Liu, S. Zhang, and Y. M. Yang, "An algorithm for automatically detecting the pulse waveform of radial artery," Beijing Biomedical Engineering, vol. 25, no. 1, 2006 (Chinese).

[10] P. H. Tsui, L. Y. Lin, C. C. Chang, et al., "Arterial pulse waveform analysis bya the probability distribution of amplitude," Physiological Measurement, vol. 28, no. 8, pp. 803-812, 2007.

[11] X. J. Huang, X. Wu, and F. Li, "Application of syntactic pattern recognition in research on pulse wave's characteristic information," Chinese Journal of Medical Instrumentation, vol. 29, no. 5, 2005 (Chinese).

[12] I. Daubechies, Ten Lectures on Wavelets, SIAM, Philadelphia, $\mathrm{Pa}, \mathrm{USA}, 1992$.

[13] S. Mallat and W. L. Hwang, "Singularity detection and processing with wavelets," IEEE Transactions on Information Theory, vol. 38, no. 2, pp. 617-643, 1992.

[14] L. J. Yang, Z. C. Song, S. P. Wang, and L. Chen, "Pulse signal identification based on wavelet transform and the fractal theory," Techniques of Automation and Applications, vol. 25, no. 7, pp. 9-12, 2006.

[15] L. Q. Zhang, Y. Li, and X. J. Zhang, "A method to extract the feature of the pulse signal based on the wavelet packet," Beijing Biomedical Engineering, vol. 24, no. 3, pp. 214-217, 2005.

[16] Y. Wang, T. Bai, and J. L. Kang, "Acquisition of pulse characteritics based on wavelet modulus maximum principle," Space Medicine \& Medical Engineering, vol. 19, no. 1, pp. 41-46, 2006.

[17] P. P. Yue and X. M. Li, "Pulse signal feature extraction and analysis based on wavelet transform," Chinese Medical Equipment Journal, vol. 27, no. 1, pp. 23-25, 2006.

[18] Y. W. Li, Y. P. Jing, T. Zheng, and Z. K. Yan, "Pulse wave signal denoisng based on the wavelet transform," Journal of Hebei University of Technology, vol. 34, no. 4, 2005 (Chinese).

[19] L. Xu, D. Zhang, and K. Wang, "Wavelet-based cascaded adaptive filter for removing baseline drift in pulse waveforms," IEEE Transactions on Biomedical Engineering, vol. 52, no. 11, pp. 1973-1975, 2005.

[20] L. Xu, D. Zhang, K. Wang, N. Li, and X. Wang, "Baseline wander correction in pulse waveforms using wavelet-based cascaded adaptive filter," Computers in Biology and Medicine, vol. 37, no. 5, pp. 716-731, 2007.

[21] K. C. Charles, An introduction to wavelets, vol. 1 of Wavelet Analysis and its Applications, Academic Press, New York, USA, 1992.

[22] Z. Nenadic and J. W. Burdick, "Spike detection using the continuous wavelet transform," IEEE Transactions on Biomedical Engineering, vol. 52, no. 1, pp. 74-87, 2005.

[23] G. Heidemann, "The long-range saliency of edge- and cornerbased salient points," IEEE Transactions on Image Processing, vol. 14, no. 11, pp. 1701-1706, 2005.

[24] A. Quddus and M. M. Fahmy, "Improved wavelet-based corner detection technique," in Proceedings of IEEE International Conference on Acoustics, Speech and Signal Processing (ICASSP '99), vol. 6, pp. 3213-3216, Phoenix, Ariz, USA, March 1999.

[25] F. A. Cheikh, A. Quddus, and M. Gabbouj, "Multi-level shape recognition based on wavelet-transform modulus maxima," in Proceedings of the 4th IEEE Southwest Symposium on Image Analysis and Interpretation (SSIAI '00), pp. 8-12, Austin, Tex, USA, April 2000.

[26] C.-L. Tu, W.-L. Hwang, and J. Ho, "Analysis of singularities from modulus maxima of complex wavelets," IEEE Transac- tions on Information Theory, vol. 51, no. 3, pp. 1049-1062, 2005.

[27] M. Penedo, W. A. Pearlman, P. G. Tahoces, M. Souto, and J. J. Vidal, "Region-based wavelet coding methods for digital mammography," IEEE Transactions on Medical Imaging, vol. 22, no. 10, pp. 1288-1296, 2003. 